\title{
Kinetic theory of Jean instability in Eddington-inspired Born-Infeld gravity
}

\author{
Ivan De Martino ${ }^{1, a}$ (D), Antonio Capolupo ${ }^{2, b}$ (D) \\ ${ }^{1}$ Department of Theoretical Physics and History of Science, Faculty of Science and Technology, University of the Basque Country UPV/EHU, \\ Barrio Sarriena s/n, 48940 Leioa, Spain \\ ${ }^{2}$ Dipartimento di Fisica E.R. Caianiello, INFN Gruppo Collegato di Salerno, Universitá di Salerno, 84084 Fisciano, SA, Italy
}

Received: 24 September 2017 / Accepted: 12 October 2017 / Published online: 26 October 2017

(C) The Author(s) 2017. This article is an open access publication

\begin{abstract}
We analyze the stability of self-gravitating systems which dynamics is investigated using the collisionless Boltzmann equation, and the modified Poisson equation of Eddington-inspired Born-Infield gravity. These equations provide a description of the Jeans paradigm used to determine the critical scale above which such systems collapse. At equilibrium, the systems are described using the time-independent Maxwell-Boltzmann distribution function $f_{0}(v)$. Considering small perturbations to this equilibrium state, we obtain a modified dispersion relation, and we find a new characteristic scale length. Our results indicate that the dynamics of self-gravitating astrophysical systems can be fully addressed in the Eddington-inspired Born-Infeld gravity. The latter modifies the Jeans instability in high densities environments, while its effects become negligible in star formation regions.
\end{abstract}

\section{Introduction}

In General Relativity (GR), matter is minimally coupled with the metric and the Einstein-Hilbert Lagrangian, which is linear in the Ricci scalar, gives rise to second order field equations. These are able to explain the dynamics of the particles up to a solar system scale, but they fail at scales of galaxies and beyond. The dynamics of self-gravitating systems and the current period of accelerated expansion of the Universe cannot be explained by just baryonic matter. Thus, GR needs to incorporate two unknown components to explain the dynamics at both galactic/extragalactic and cosmological scales. Specifically, almost $\sim 68 \%$ of the total amount of the matter and energy in the Universe should be in form of the cosmological constant, or more in general of dark energy,

\footnotetext{
a e-mail: ivan.demartino@ehu.eus

b e-mail: capolupo@sa.infn.it
}

while $\sim 26 \%$ should be in the form of invisible and exotic particles, named dark matter. Nevertheless their fundamental nature is still unknown [1-4]. The need to incorporate them has been interpreted as a breakdown of GR at astrophysical and cosmological scales, opening the door to alternative theories of gravity.

Generalizations of the gravitational action have been extensively explored to overcome the need of these two exotic components. On th one hand these are motivated by their capability to explain the dynamics of self-gravitating systems and the accelerated expansion of the Universe without resorting to dark matter and/or dark energy [5-14]. On the other hand, they are also motivated by the fact that GR is not the quantum theory of gravity needed to describe the spacetime near the singularities, which, as is well known, seemingly cannot be avoided [15]. Although a quantum theory of gravity should be able to overcome such problems, there also exists the possibility to avoid singularities modifying the coupling between matter and gravity. In this context, Eddingtoninspired Born-Infeld (EiBI) gravity has been recently proposed [16]. EiBI gravity is inspired by the Born-Infeld action for non-linear electrodynamics, with the Ricci tensor replacing the field tensor $F_{\mu \nu}$. This structure was motived by some classes of string theories where the Born-Infeld electrodynamics arises as a low-energy effective theory $[17,18]$. One of the most interesting features is that EiBI is equivalent to GR in the vacuum while it introduces modifications in dense matter environments, where GR is experimentally not well probed. EiBI is able to describe, with only a single extra parameter $(\kappa)$, astrophysical objects such as the Sun [19] and the internal structure of compact objects [20-26], and the cosmological expansion of the Universe [22,27-31] (for comprehensive reviews see $[7,12]$ and the references therein).

Briefly, the gravitational action of EiBI gravity takes the following form: 
$S=\frac{2}{\kappa} \int \mathrm{d}^{4} x\left(\sqrt{\left|g_{\mu \nu}+\kappa R_{\mu \nu}\right|}-\lambda \sqrt{-g}\right)+S_{\text {matter }}\left[g, \phi_{M}\right]$,

where $R_{\mu \nu}$ is the symmetric part of the Ricci tensor, $\phi_{M}$ represents the matter field, and $\lambda$ is a constant. The latter is linked to the cosmological constant in such a way that one obtains asymptotically flat solutions setting $\lambda=1$. Finally, the field equations are built varying the action as in the Palatini approach. As in other modified theories of gravity, the Palatini approach is not equivalent to a pure metric one. However, the latter contains ghosts that can be eliminated only adding extra terms in the gravitational action $[32,33]$. The higher order curvature terms account for both non-linear matter coupling and for avoiding singularities. Such correction terms also appear in the non-relativistic limit, where EiBI gravity leads to a modified Poisson equation given by

$\nabla^{2} \Phi(\mathbf{r}, t)=4 \pi G \rho(\mathbf{r}, t)+\frac{\kappa}{4} \nabla^{2} \rho(\mathbf{r}, t)$,

where $\Phi(\mathbf{r}, t)$ is the gravitational potential, and $\rho(\mathbf{r}, t)$ is the matter density. Let us note that by setting $\kappa=0$, the previous equation immediately reduces to the standard Poisson equation $\nabla^{2} \Phi(\mathbf{r}, t)=4 \pi G \rho(\mathbf{r}, t)$. The tightest constraint in the literature on the EiBI parameter has been obtained comparing the electromagnetic and gravitational interactions inside atomic nuclei: $|\kappa|<10^{-3} \mathrm{~kg}^{-1} \mathrm{~m}^{5} \mathrm{~s}^{-2}$ [37].

In this paper, we analyze the kinetic theory of the Jean instability for self-gravitating systems in EiBI gravity. This mechanism constitutes, on the theoretical side, a remarkable instrument to retain/rule out modified theories of gravity at astrophysical level. In fact, although a self-gravitating system collapses under the gravitational force induced by the modified Poisson equation and gives rise to star formation, we expect that the effects of the EiBI gravity are totally negligible in star formation environments while they must show some deviations from GR in compact objects collapsing into Black Holes.

The paper is organized as follows: in Sect. 2 we compute the dispersion relation for an homogeneous self-gravitating system in EiBI gravity; in Sect. 3 we analyze the dispersion relation in low and high frequency regimes, and we study the unstable modes that led to the collapse of the structure; in Sect. 4 we give our conclusion and remarks.

\section{Dispersion relation of a collisionless self-gravitating system}

The standard approach to describing the collapse of a selfgravitating system, either a star formation regions such as interstellar clouds which physical conditions change from hot X-ray emitting plasma to cold molecular gas, or compact objects collapsing into Black Hole, is the Jeans instability [34]. The latter is usually described by a distribution function of the particles $f(\mathbf{r}, \mathbf{v}, t)$ which is solution of the BoltzmanVlasov system of equations:

$$
\begin{aligned}
& {\left[\frac{\partial}{\partial t}+\left(\mathbf{v} \cdot \nabla_{r}\right)-\left(\nabla \Phi(\mathbf{r}, t) \cdot \nabla_{v}\right)\right] f(\mathbf{r}, \mathbf{v}, t)=I_{\mathrm{coll}},} \\
& \nabla^{2} \Phi(\mathbf{r}, t)=\left[4 \pi G+\frac{\kappa}{4} \nabla^{2}\right] \rho(\mathbf{r}, t)
\end{aligned}
$$

where the mass density distribution reads

$\rho(\mathbf{r}, t)=\int f(\mathbf{r}, \mathbf{v}, t) \mathrm{d} \mathbf{v}$.

Here $I_{\text {coll }}$ is the collision term and, since we analyze a collisionless system, it can be neglected $\left(I_{\text {coll }}=0\right)$.

To study the effect of the EiBI gravity, Eqs. (3)-(5) must be linearized. Assuming that the unperturbed potential $\Phi_{0}$ is locally constant in the system, one can set $\nabla \Phi_{0}=0$. This is generally known as Jeans swindle [34]. Thus, in small perturbations regime, the distribution function and the gravitational potential can be written as

$$
\begin{aligned}
& f(\mathbf{r}, \mathbf{v}, t)=f_{0}(\mathbf{r}, \mathbf{v})+\varepsilon f_{1}(\mathbf{r}, \mathbf{v}, t) \\
& \Phi(\mathbf{r}, t)=\Phi_{0}(\mathbf{r})+\varepsilon \Phi_{1}(\mathbf{r}, t)
\end{aligned}
$$

where $\varepsilon \ll 1$ for small perturbations. Thus, at the first order, the Boltzman-Vlasov system of equations can be recast in the Fourier space as follows:

$$
\begin{aligned}
& -i \omega f_{1}+\mathbf{v} \cdot\left(i \mathbf{k} f_{1}\right)-\left(i \mathbf{k} \Phi_{1}\right) \cdot \frac{\partial f_{0}}{\partial \mathbf{v}}=0 \\
& -k^{2} \Phi_{1}=4 \pi G \int f_{1}(\mathbf{r}, \mathbf{v}, t) \mathrm{d} \mathbf{v}-\frac{\kappa}{4} k^{2} \int f_{1}(\mathbf{r}, \mathbf{v}, t) \mathrm{d} \mathbf{v}
\end{aligned}
$$

and the dispersion relation reads

$1+\left(\frac{4 \pi G}{k^{2}}-\frac{\kappa}{4}\right) \int \frac{\mathbf{k} \cdot \frac{\partial f_{0}}{\partial \mathbf{v}}}{\mathbf{v} \cdot \mathbf{k}-\omega} \mathrm{d} \mathbf{v}=0$

Assuming the local thermodynamical equilibrium, the background distribution function of the particles can be described using the Maxwell-Boltzman distribution. Therefore, $f_{0}(\mathbf{v})$ is given by

$$
f_{0}(\mathbf{v})=\frac{\rho_{0}}{\left(2 \pi \sigma^{2}\right)^{\frac{3}{2}}} \mathrm{e}^{-\frac{v^{2}}{2 \sigma^{2}}},
$$

where $\rho_{0}$ is the matter density at equilibrium, and $\sigma$ is the thermal dispersion velocity of the particles. Finally, inserting 
the Maxwell-Boltzman distribution in Eq. (10) the dispersion relation reads

$1-\left(\frac{4 \pi G}{k^{2}}-\frac{\kappa}{4}\right) \frac{\rho_{0}}{\sqrt{2 \pi} \sigma^{3}} k \int \frac{v \mathrm{e}^{-\frac{v^{2}}{2 \sigma^{2}}}}{k v-\omega} \mathrm{d} v=0$.

The previous equation shows a singularity at $\omega=k v$. Moreover, setting $\kappa=0$, it reduces to the Newtonian dispersion relation. Thus, one can infer the limit for the collapse setting $\omega=0$ and computing the maximum wavelength of the perturbations supported by the system, and above which the system collapses. Specifically, in the Newtonian case, setting $\omega=0$ one obtains the so-called Jeans wavenumber

$k_{J}^{2}=\frac{4 \pi G \rho_{0}}{\sigma^{2}}$,

that can be used to define the Jeans mass as the mass enclosed in a sphere of radius $\lambda_{J}=2 \pi / k_{J}$, obtaining

$$
M_{J}=\frac{\pi}{6} \sqrt{\frac{1}{\rho_{0}}\left(\frac{\pi \sigma^{2}}{G}\right)}
$$

On the one hand, perturbations having wavelength $\lambda>\lambda_{J}$ are unstable, and they exponentially grow. On the other hand, if the wavelength is less than the Jeans limit then perturbations are strongly damped. In EiBI gravity, such limit as a result is modified by the additional term in the Poisson equation. Therefore, from Eq. (12) we obtain

$k^{* 2}(\omega=0)=\left(\frac{\sigma^{2}}{4 \pi G \rho 0}+\frac{\kappa}{16 \pi G}\right)^{-1}=\left(1+\frac{\kappa}{16 \pi G} k_{J}^{2}\right)^{-1} k_{J}^{2}$,

which corresponds to a mass

$M^{*}=\frac{\pi}{6} \sqrt{\frac{1}{\rho_{0}}\left(\frac{\pi \sigma^{2}}{G}+\frac{4 \pi^{2} \rho_{0} \kappa}{16 \pi G}\right)^{3}}=\left(1+k_{J}^{2} \frac{\kappa}{16 \pi G}\right)^{3 / 2} M_{J}$.

Thus, the mass limit for the collapse of a self-gravitating system depends on the EiBI parameter. It can be higher or lower than the classical Jeans Mass and, as a consequence, EiBI gravity can favor or disfavor the gravitational collapse depending on the sign of $\kappa$.

\section{Analysis of the collisionless dispersion relation}

Equation (12) can easily be rewritten as

$$
1-\left(\frac{k_{J}^{2}}{k^{2}}-\frac{\kappa}{16 \pi G} k_{J}^{2}\right) \frac{1}{\sqrt{2 \pi}} \int \frac{x \mathrm{e}^{-\frac{x^{2}}{2}}}{\beta-x} \mathrm{~d} x=0,
$$

where we have defined the following variables:

$\beta=\frac{\omega}{k \sigma} ; \quad x=\frac{v_{x}}{\sigma}$.

Moreover, in EiBI gravity there naturally arises a new wavelength

$\lambda_{E i B I}=\sqrt{\frac{\pi|\kappa|}{4 G}}$,

which is equal to the one found at cosmological scale and using fluid approach [22]. The EiBI wavelength allows us to rewrite the dispersion relation in a more compact form

$1-\left(\frac{k_{J}^{2}}{k^{2}}-\frac{k_{J}^{2}}{k_{\mathrm{EiBI}}^{2}}\right) \frac{1}{\sqrt{2 \pi}} \int \frac{x \mathrm{e}^{-\frac{x^{2}}{2}}}{\beta-x} \mathrm{~d} x=0$

Let us study the limit of high frequency perturbations $\beta \gg 1$.

In this case, having no singularities, we can integrate Eq. (20) along the real axis $\left(\omega=\omega_{R}+i \omega_{I} \approx \omega_{R}\right)$ obtaining

$1+\frac{3 k^{2} k_{J}^{2} \sigma^{4}}{\omega_{R}^{4}}-\frac{3 k^{4} k_{J}^{2} \sigma^{4}}{k_{\mathrm{EiBI}}^{2} \omega_{r}^{4}}+\frac{k_{J}^{2} \sigma^{2}}{\omega_{R}^{2}}-\frac{k^{2} k_{J}^{2} \sigma^{2}}{k_{\mathrm{EiBI}}^{2} \omega_{R}^{2}}=0$,

which is a quadratic equation for $\omega_{R}^{2}$. Remembering the condition $\beta \gg 1$, we find

$\omega_{R}^{2}=\left[k^{2}\left(3+\frac{k_{J}^{2}}{k_{\mathrm{EiBI}}^{2}}\right)-k_{J}^{2}\right] \sigma^{2}$,

which for $\kappa=0$ reduces to the classical Newtonian relation. Thus, as for the Newtonian case, high frequency perturbations with $k^{2}>k_{J}\left(\frac{k_{\mathrm{EiBI}}^{2}}{3 k_{\mathrm{EiBI}}^{2}+k_{J}^{2}}\right)$ are quickly damped by the self-gravitating system whenever the EiBI wavenumber satisfies the condition $k_{\mathrm{EiBI}}^{2}>-k_{J}^{2} / 2$. On the contrary, high frequency perturbations can be supported by the system as is shown in Fig. 1 (magenta line).

More interesting is to consider the case of low frequency perturbations $\beta \ll 1$, which in the Newtonian case led to unstable modes. In such a limit, the integral in Eq. (20) can be recast into the form

$\frac{1}{\sqrt{2 \pi}} \int \frac{x \mathrm{e}^{-\frac{x^{2}}{2}}}{\beta-x} \mathrm{~d} x \approx 1+\imath \sqrt{\frac{\pi}{2}} \beta$.

Therefore, splitting $\omega$ in its real and imaginary part, $\omega=$ $\omega_{R}+\imath \omega_{I}$, and setting $\omega_{R}=0$, we find

$\omega_{I}=k \sigma \sqrt{\frac{2}{\pi}}\left[1-\left(\frac{k_{J}^{2}}{k^{2}}-\frac{k_{J}^{2}}{k_{\mathrm{EiBI}}^{2}}\right)^{-1}\right]$, 


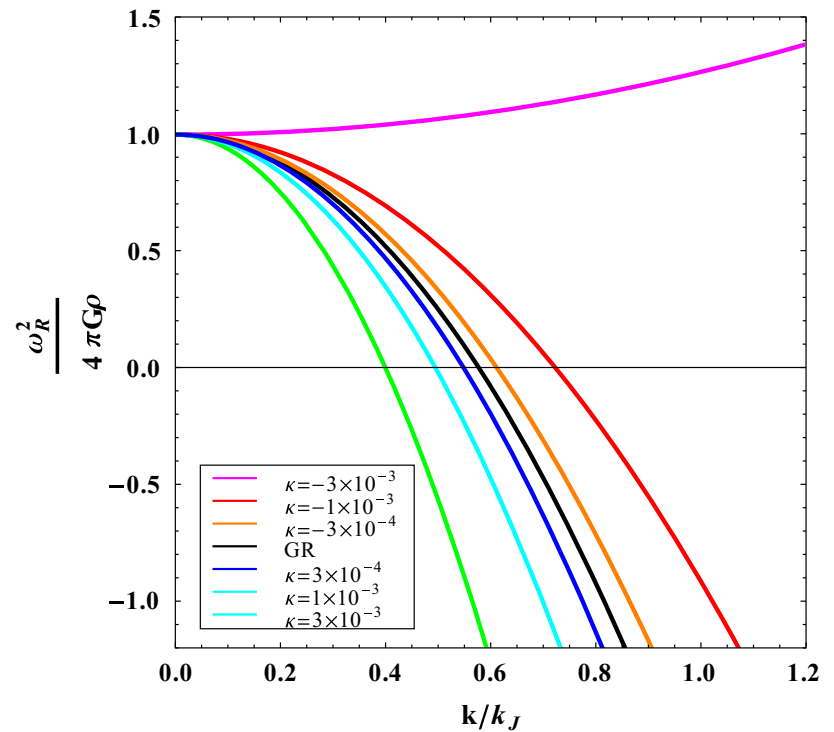

Fig. 1 High frequency limit of growth rate of the Jeans instability. The oscillations of the plasma are quickly damped (as for the Newtonian case)

which gives unstable modes when $\omega_{I}>0$, that is, for

$\left(\frac{k_{J}^{2}}{k^{2}}-\frac{k_{J}^{2}}{k_{\mathrm{EiBI}}^{2}}\right)^{-1}<1$

In other words, the system supports perturbations having a wavelength

$\lambda^{2}>\lambda^{* 2} \equiv \lambda_{J}^{2}-\lambda_{\mathrm{EiBI}}^{2}$,

while it shows a singularity for perturbations having $\lambda=$ $\lambda_{\text {EiBI }}$. Perturbations having $\lambda<\lambda^{*}$ are quickly damped by the system staying stable. Next, while in Newtonian gravity perturbations having $\lambda>\lambda_{J}$ we are capable to generate the collapse of the system, in EiBI gravity the limit for the collapse becoming lower or higher depending on the parameter $\kappa$. This can be quickly understood looking at Eq. (16). Since the EiBI modification depends on the coupling between matter and gravity, which is larger as the density increases [35], its effects must be negligible in low density environments like star formation regions where the condition $\lambda_{\mathrm{EiBI}} \ll \lambda_{J}$ must hold. This condition can be straightforwardly translated into a density threshold,

$\rho_{0}<\rho_{0}^{*}=\frac{\sigma^{2}}{\pi^{2} \kappa}$

which means that EiBI gravity does not affect systems having density below $\rho_{0}^{*}$. On the one hand, the interstellar medium has temperatures ranging from 10 to $100 \mathrm{~K}$, while matter density ranges from $\rho_{0} \sim 10^{-18}$ to $\rho_{0} \sim 10^{-16} \mathrm{~kg} \mathrm{~m}^{-3}$. Setting $|\kappa|<10^{-3} \mathrm{~kg}^{-1} \mathrm{~m}^{5} \mathrm{~s}^{-2}$ [37] it follows that the density

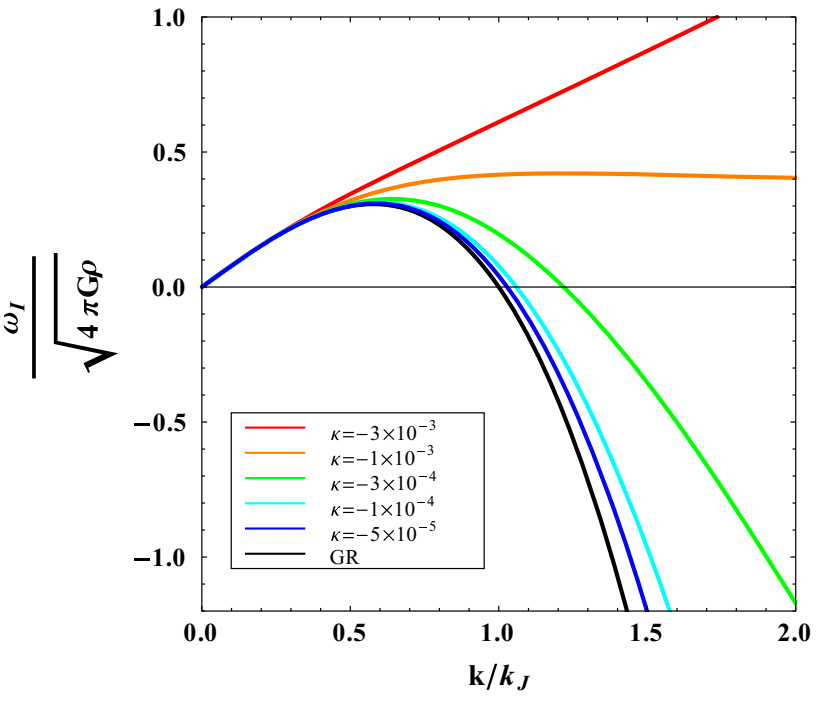

Fig. 2 The growth rate of the Jeans instability versus $k / k_{J}$ for different values of the EiBI parameter. Here the dispersion relation is illustrated for the cases corresponding to $\kappa<0$

threshold is $\rho_{0}^{*} \approx 10^{7} \mathrm{~kg} \mathrm{~m}^{-3}$. As expected, $\rho_{0}<\rho_{0}^{*}$ in a star formation region. Therefore, EiBI gravity can be neglected. On the other hand, high density and temperature systems such as a Hyper Massive Neutron Star (HMNS, [36]) are one of the most promising laboratories to probe EiBI gravity. Those systems arise from the merger of a neutron star binary, and they are expected to collapse to a rotating black hole having a massive accretion torus. The temperature of those systems lies in the range $[5,50] \times 10^{10} \mathrm{~K}$ with a particle number density $\sim 10^{39} \mathrm{~cm}^{-3}$. The collapse of such a system is considered as one of the possible sources of short gamma ray bursts. For such systems, with $\rho_{0}^{*} \approx 10^{15} \mathrm{~kg} \mathrm{~m}^{-3}$ and $\rho_{0} \approx 10^{18} \mathrm{~kg} \mathrm{~m}^{-3}$, EiBI gravity affects the kinetic instability producing deviations from the GR.

Figures 2 and 3 display the growth rate of the unstable roots of Eq. (24) as a function of the normalized wavenumber $k / k_{J}$ and for different values of the EiBI parameter $\kappa$. Specifically, Fig. 2 illustrates the growth rate for a few cases corresponding to negative values of $\kappa$, while Fig. 3 is devoted to positive values of $\kappa$. Figure 2 illustrates that the growth rate is larger for lower values of $\kappa$, while as $\kappa$ is closer to zero (which corresponds to the Newtonian solution) the system shows unstable modes for $k<k^{*}$, and stable modes for $k>k^{*}$. Nevertheless, the Jeans mass as a result is higher (see Eq. (16)); therefore the collapse of the HMNS is disfavored with respect to the Newtonian case.

More interesting is the growth rate for positive values of $\kappa$ shown in Fig. 3. In this case the dispersion relation has a singularity at $k=k_{\mathrm{EiBI}}$. The physical behavior around such a point can only be described by more complex physical theories where a singularity does not occur. Therefore, the study here presented shows also the limit of the Jeans instability in 


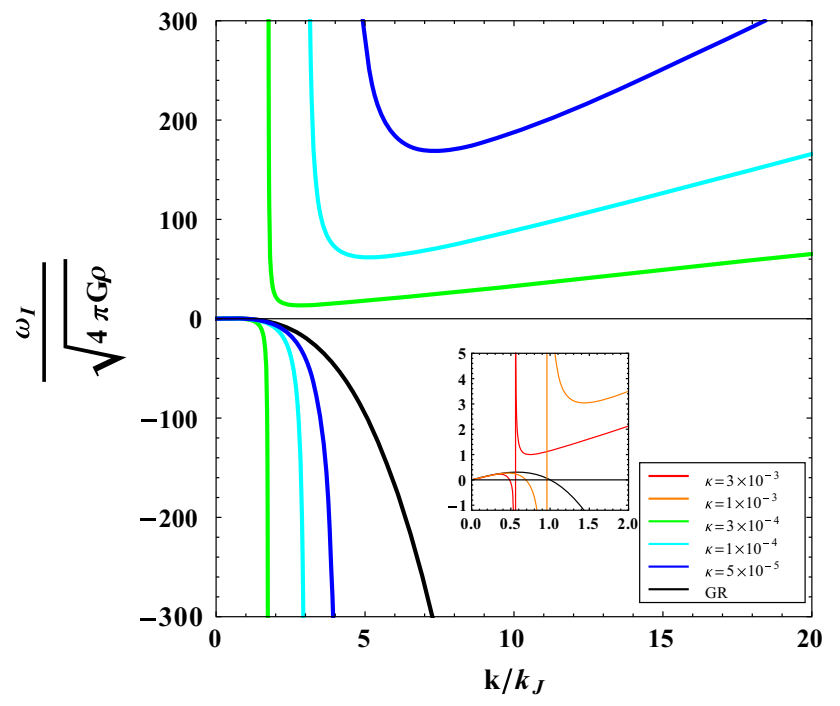

Fig. 3 The growth rate of the Jeans instability versus $k / k_{J}$ for different values of the EiBI parameter. Here the dispersion relation is illustrated for the cases corresponding to $\kappa>0$

EiBI gravity, which cannot be blindly applied in the points around $k=k_{\mathrm{EiBI}}$.

Notice that such dramatic changes in $\omega_{I}$ correspond to a phase transition of the system. In thermodynamic systems, phase transitions occur when the free energy, or the partition function, has singularities for some choice of variables, such as the temperature. For example, in the phenomenon of superconductivity, certain materials, when cooled below a critical temperature $T_{C}$, pass into the superconducting state characterized by zero electrical resistance and by the complete ejection of magnetic field lines from the interior of the superconductor. In other systems, other physical parameters play the role of the temperature. For example, quantum phase transitions can be obtained by varying the magnetic field or the pressure at zero temperature.

In our case, a significant physical parameter is the wavenumber $k$ and the critical value of $k$, which marks the phase transition is $k_{\mathrm{EiBI}}$. Indeed, waves having wavenumbers smaller than $k_{\mathrm{EiBI}}$ and satisfying the condition $k<k^{*}$ give rise to unstable modes that favor the collapse of the HMNS into a black hole. Meanwhile, waves having wavenumber higher than the EiBI wavenumber show only stable solutions that do not produce the collapse of the structure even for $k_{\mathrm{EiBI}}<k<k^{*}$.

We further remark that the extra modes $k \sim k_{\mathrm{EiBI}}$ do not exist in Newtonian gravity where the singularity in $k=k_{\mathrm{EiBI}}$ is absent in such a model (see Fig. 3), and also that this mode does not depend on the approximation made to compute the dispersion relation in Eq. (24) since it is straightforward to highlight that such a singularity is also present in the general expression of the dispersion relation given in Eq. (10). Therefore, this sort of phase transition could be an indication that the general paradigm of the Jeans instability, which works fine in GR and in other modified gravity models [38], does not work anymore in EiBI gravity for $k>k_{\mathrm{EiBI}}$.

\section{Conclusions and remarks}

We have investigated the impact of the recently proposed EiBI gravity theory on the gravitational collapse of a selfgravitating system. We have solved the collisionless Boltzmann equation together with the modified Poisson equation of EiBI gravity to study the kinetic instability of selfgravitating system, and we have computed the corresponding dispersion relation leading to a new gravitational scale length. We studied both the high and low frequency limits of the dispersion relation. In the high frequency limit, the self-gravitating system behaves as in Newtonian gravity, not supporting the propagation of the perturbations. In the low frequency limit EiBI gravity may introduce a modification to the Jeans instability. Although EiBI gravity modified the Jeans mass needed for the collapse, it does not affect the star formation because of the low density environments where it happens. Nevertheless, in higher density environments such as HMNS the higher order terms of EiBI gravity produce a departure from the Newtonian growth rate, which could be both an indication that the standard Jeans paradigm does not hold anymore, or that effective new modes are present that could serve as a mechanism to generate a black hole from massive stars.

Acknowledgements IDM acknowledges financial supports from University of the Basque Country UPV/EHU under the program "Convocatoria de contratación para la especialización de personal investigador doctor en la UPV/EHU 2015", and from the Spanish Ministerio de Economía y Competitividad through the research project FIS201015492, and from the Basque Government through the research project IT-956-16. AC acknowledges partial financial support from MIUR and INFN. The authors acknowledges the COST Action CA1511 Cosmology and Astrophysics Network for Theoretical Advances and Training Actions (CANTATA).

Open Access This article is distributed under the terms of the Creative Commons Attribution 4.0 International License (http://creativecomm ons.org/licenses/by/4.0/), which permits unrestricted use, distribution, and reproduction in any medium, provided you give appropriate credit to the original author(s) and the source, provide a link to the Creative Commons license, and indicate if changes were made.

Funded by $\mathrm{SCOAP}^{3}$.

\section{References}

1. Planck Collaboration, A\&A 594, A13 (2016)

2. J.L. Feng, Ann. Rev. Astron. Astrophys. 48, 495 (2010)

3. M. Yu, Khlopov Mod. Phys. Lett. A 32, 1702001 (2017)

4. A. Capolupo, Adv. High Energy Phys. 2016, 8089142 (2016)

5. S. Capozziello, M. De Laurentis, Phys. Rep. 509, 167 (2011) 
6. S. Capozziello, M. De Laurentis, Ann. Phys. 524, 545 (2012)

7. T. Clifton, P.G. Ferreira, A. Padilla, C. Skordis, Phys. Rep. 513, 1 (2012)

8. I. de Martino, M. De Laurentis, F. Atrio-Barandela, S. Capozziello, MNRAS 442(2), 921-928 (2014)

9. I. de Martino, M. De Laurentis, S. Capozziello, Universe 1, 123 (2015)

10. Y.-F. Cai, S. Capozziello, M. De Laurentis, E.N. Saridakis, Rep. Prog. Phys. 79(4), 106901 (2016)

11. I. de Martino, Phys. Rev. D 93, 124043 (2016)

12. J. Beltran Jimenez, L. Heisenberg, G.J. Olmo, D. Rubiera-Garcia (2017). arXiv:1704.03351

13. S. Nojiri, S.D. Odintsov, Phys. Rept. 505, 59-144 (2011). https:// doi.org/10.1016/j.physrep.2011.04.001

14. S. Nojiri, S.D. Odintsov, V.K. Oikonomou, Phys. Rept. 692, 1-104 (2017). https://doi.org/10.1016/j.physrep.2017.06. 001. arXiv:1705.11098

15. S.W. Hawking, G.F.R. Ellis, The Large Scale Structure of SpaceTime (Cambridge University Press, Cambridge, 1973)

16. M. Banados, P.G. Ferreira, Phys. Rev. Lett. 105, 011101 (2010)

17. M. Born, L. Infeld, Proc. R. Soc. A 144, 425-451 (1934)

18. E. Fradkin, A.A. Tseytlin, Phys. Lett. B 163, 123 (1985)

19. J. Casanellas, P. Pani, I. Lopes, V. Cardoso, Astrophys. J. 745, 15 (2012)

20. P. Pani, V. Cardoso, T. Delsate, Phys. Rev. Lett. 107, 031101 (2011)

21. P. Pani, T. Delsate, V. Cardoso, Phys. Rev. D 85, 084020 (2012)

22. P.P. Avelino, Phys. Rev. D 85, 104053 (2012)
23. Y.-H. Sham, L.-M. Lin, P.T. Leung, Phys. Rev. D 86, 064015 (2012)

24. Y.-H. Sham, P.T. Leung, L.-M. Lin, Phys. Rev. D 87, 061503(R) (2013)

25. T. Harko, F.S.N. Lobo, M.K. Mak, S.V. Sushkov, Phys. Rev. D 88, 044032 (2013)

26. H. Sotami, Phys. Rev. D 89, 104005 (2014)

27. P.P. Avelino, R.Z. Ferreira, Phys. Rev. D 86, 041501 (2012)

28. A. De Felice, B. Gumjudpai, S. Jhingan, Phys. Rev. D 86, 043525 (2012)

29. M. Bouhmadi-López, I. Albarran, C.-Y. Chen, Universe 3(2), 36 (2017)

30. I. Albarran, M. Bouhmadi-López, C.-Y. Chen, P. Chen, Phys. Lett. B 772, 814-818 (2017)

31. M. Bouhmadi-López, C.-Y. Chen, P. Chen, Eur. Phys. J. C 75, 90 (2015)

32. S. Deser, G.W. Gibbons, Class. Quantum Gravity 15, L35 (1998)

33. D.N. Vollick, Phys. Rev. D 69, 064030 (2004)

34. J. Binney, S. Tremaine, Galactic Dynamics (Princeton University Press, Princeton, 1994)

35. T. Delsate, J. Steinhoff, Phys. Rev. Lett. 109, 021101 (2012)

36. L. Rezzolla, O. Zanotti, Relativistic Hydrodynamics (Oxford University Press, Oxford, 2013)

37. P.P. Avelino, JCAP 11, 022 (2012)

38. S. Capozziello, M. de Laurentis, I. de Martino, M. Formisano, S.D. Odintsov, Phys. Rev. D 85(4), 044022 (2012) 